Musées, Patrimoine et Culture scientifiques et techniques

$188 \mid 2020$

mars-avril 2020

\title{
Le Palais de la découverte 2024 : réflexions sur un projet
}

\section{Bruno Maquart}

\section{OpenEdition}

Journals

Édition électronique

URL : https://journals.openedition.org/ocim/3687

DOI : 10.4000/ocim.3687

ISSN : 2108-646X

Éditeur

OCIM

Édition imprimée

Date de publication : 1 mars 2020

Pagination : $38-43$

ISSN : 0994-1908

Référence électronique

Bruno Maquart, «Le Palais de la découverte 2024 : réflexions sur un projet », La Lettre de l'OCIM [En

ligne], 188 | 2020, mis en ligne le 01 mars 2021, consulté le 17 juillet 2021. URL : http://

journals.openedition.org/ocim/3687 ; DOI : https://doi.org/10.4000/ocim.3687

Ce document a été généré automatiquement le 17 juillet 2021.

Tous droits réservés 


\section{Le Palais de la découverte 2024 : réflexions sur un projet}

\section{Bruno Maquart}

Vue d'architectedu Palais éphémère, qui ouvrira en juin 2020.

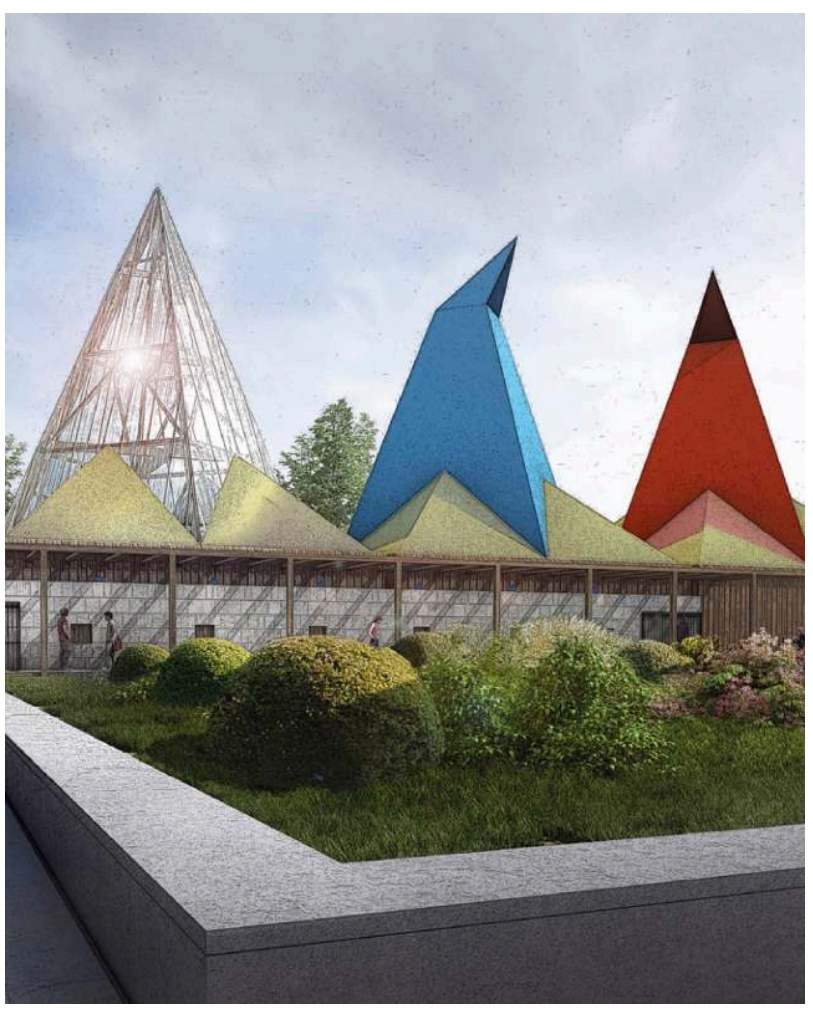

(c) A . Deswarte pour Atelier Construire

Le Palais de la découverte entamera dans quelques mois sa rénovation complète. Après une phase lancée en 2016 en site occupé (qui a vu la restauration de la rotonde d'entrée du Palais et de ses toitures), de lourds travaux vont être engagés au cœur même de 
l'édifice. Maintes fois différée, la rénovation du Palais - dont on a fêté les 80 ans en 2017 - advient enfin, après plusieurs tentatives avortées. Elle viendra ainsi, d'une certaine manière, clore le cycle des grands travaux culturels nationaux, permettant au Palais de la découverte de rejoindre, dans le cercle des établissements de culture scientifique modernisés, le musée des arts et métiers, le musée de l'Homme, le musée du Quai Branly-Jacques Chirac, ou encore le musée des Confluences à Lyon ou le musée des civilisations de l'Europe et de la Méditerranée à Marseille. L'opération, qui prendra plusieurs années, s'inscrit dans le large cadre du schéma directeur de restauration et d'aménagement du Grand Palais, engagé à la suite du rapport Cluzel remis en avril 2010 : elle concerne ainsi, selon un programme et une répartition des surfaces arrêtés en 2011, l'ensemble du bâtiment et donc, non seulement le Palais de la découverte, mais aussi la Nef du Grand Palais, les galeries nationales dans lesquelles la Réunion des musées nationaux (RMN) présente ses grandes expositions, de même que le commissariat de police logé sur son flanc Nord. L'enjeu de la restauration du monument signé des architectes Henri Deglane, Louis-Albert Louvet et Albert Thomas est de "remettre au jour la tension entre l'expression académique des dehors, la clarté de la composition spatiale et la modernité des transparences et des lumières des dedans $»^{1}$. Une fois restauré, le Grand Palais, avec le Petit Palais qui lui fait face, constituera un exemple, unique à Paris, d'architecture 1900, situé au pied de la "plus belle avenue du monde».

\section{Les origines du Palais}

Le Palais de la découverte est l'œuvre de Jean Perrin, homme politique français et prix Nobel de physique. Imaginé au départ pour être présenté temporairement lors de l'exposition internationale de Paris de 1937, le Palais rencontre un succès tel qu'il devient permanent, rouvrant au public en 1938 après quelques mois de travaux. Dès l'origine, le Palais de la découverte incarne un concept nouveau : il ne sera pas un musée voué à présenter des objets scientifiques et technologiques, mais un lieu d'innovation scientifique, qui ouvre au public les portes des laboratoires. Ici, les visiteurs verront " la science en train de se faire » au moyen d'expériences, dont certaines très spectaculaires, présentées par des scientifiques. S'adressant à tous, et particulièrement aux jeunes dans le but de susciter des vocations, il joue dès ses débuts un rôle majeur dans la vulgarisation de la science en France et à l'étranger. 
Démonstration en salle Pi du Palais de la découverte en 1937.

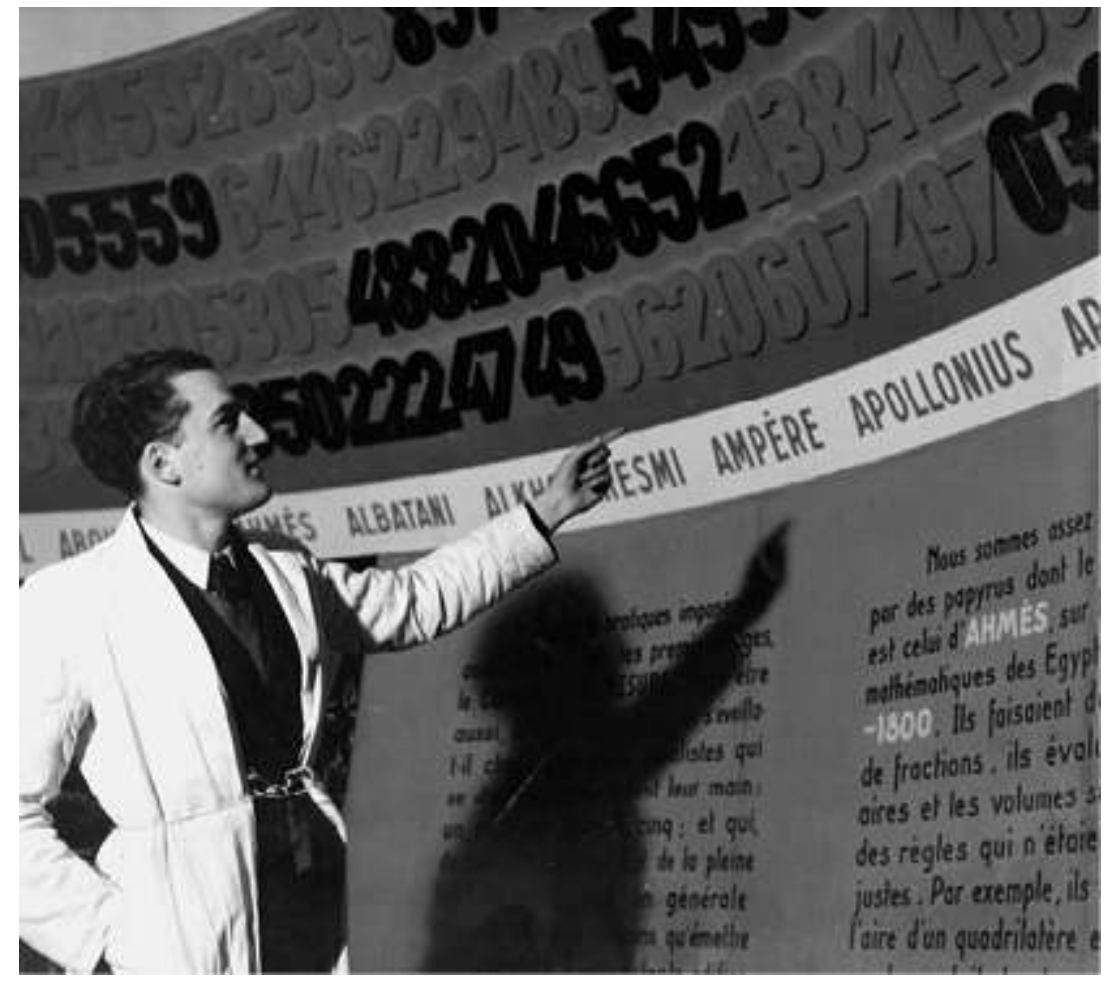

(c) Palais de la découverte/Pytlik

2 Le rapport Cluzel, sur la base duquel l'État a engagé cette opération importante et dont il a confié la maîtrise d'ouvrage à la RMN, affectataire de l'ensemble du bâtiment ${ }^{2}$, en a résumé les finalités culturelles dans l'expression de "Grand Palais des arts et des sciences». Cette expression a soulevé des interrogations, de même que le projet scientifique et culturel du Palais de la découverte $2024^{3}$, auxquelles le présent article entend répondre. L'expression "arts et sciences» doit s'entendre ainsi que ramassée dans l'équation suivante :

arts et sciences $=$ arts + sciences $+($ arts + sciences $)$

4 Autrement dit, le Grand Palais hébergera demain des activités artistiques, sous la responsabilité culturelle de la RMN, des activités scientifiques, sous celle d'Universcience dans le Palais de la découverte, ainsi que des activités hybrides, conduites par l'un, l'autre ou les deux opérateurs culturels ensemble, comme la galerie des enfants qui sera co-gérée par les deux institutions.

5 Le Palais de la découverte de demain sera à la fois proche de celui que nous connaissons aujourd'hui et sensiblement renouvelé: ni tout à fait le même, ni tout à fait un autre, pour paraphraser le poète. Conservant intact son ADN, il sera demain le musée qu'il a toujours été. La médiation humaine demeurera sa marque distinctive et les médiateurs ne seront pas remplacés par des écrans. Les exposés, présentations spectaculaires et ateliers se tiendront dans un cadre rénovét, adapté à leurs besoins spécifiques et permettant d'en maintenir le volume actuel ${ }^{5}$. De nouvelles offres et activités seront par ailleurs développées pour le public, sur une surface équivalente à celle d'aujourd'hui. Le projet de rénovation est ainsi une opportunité unique d'actualiser la proposition du Palais de la découverte, pour qu'il respecte sa vocation de parler de la science d'aujourd'hui au public d'aujourd'hui. Car nul ne contestera que tant la science que le 
public ont changé. À cet égard, Jean Perrin, dont nous fêterons cette année le $150^{\mathrm{e}}$ anniversaire, avait posé des bases qui demeurent pertinentes: «Les institutions doivent être bâties en hommes plus qu'en pierres. Un Palais permanent de la découverte ne sera utile que si, loin d'être une sorte de Musée bientôt stérilisé dans l'immobilité, il garde un contact vivant avec la Science qui continue à se créer, ceci grâce à l'effort soutenu de chercheurs et de savants qui feront de cet effort leur activité principale " ${ }^{6}$.

6 Le Palais de la découverte parlera toujours des sciences fondamentales, se distinguant en cela de la Cité des sciences et de l'industrie, qui s'intéresse aux rapports entre la science et la société, donnant à voir les innovations technologiques et industrielles. Le Palais a pour mission de montrer tous les aspects de la science moderne : la science en tant qu'ensemble de connaissances, qui offre aux visiteurs la clé des concepts fondamentaux comme celle des derniers développements scientifiques; la science en tant qu'entreprise collective, internationale et plus souvent pluridisciplinaire; la science en tant que vecteur de compréhension du monde qui nous entoure; la science comme démarche, qui s'appuie sur des faits plutôt que de s'incliner devant l'autorité, et rend capable de contrer les «infox». Il ajoutera ainsi, à la transmission des fondamentaux des sciences, un programme permanent, approfondi et de plus grande ampleur sur la recherche contemporaine et les questions auxquelles elle s'attache à répondre.

Médiation de chimie au Palais de la découverte - actuellement.

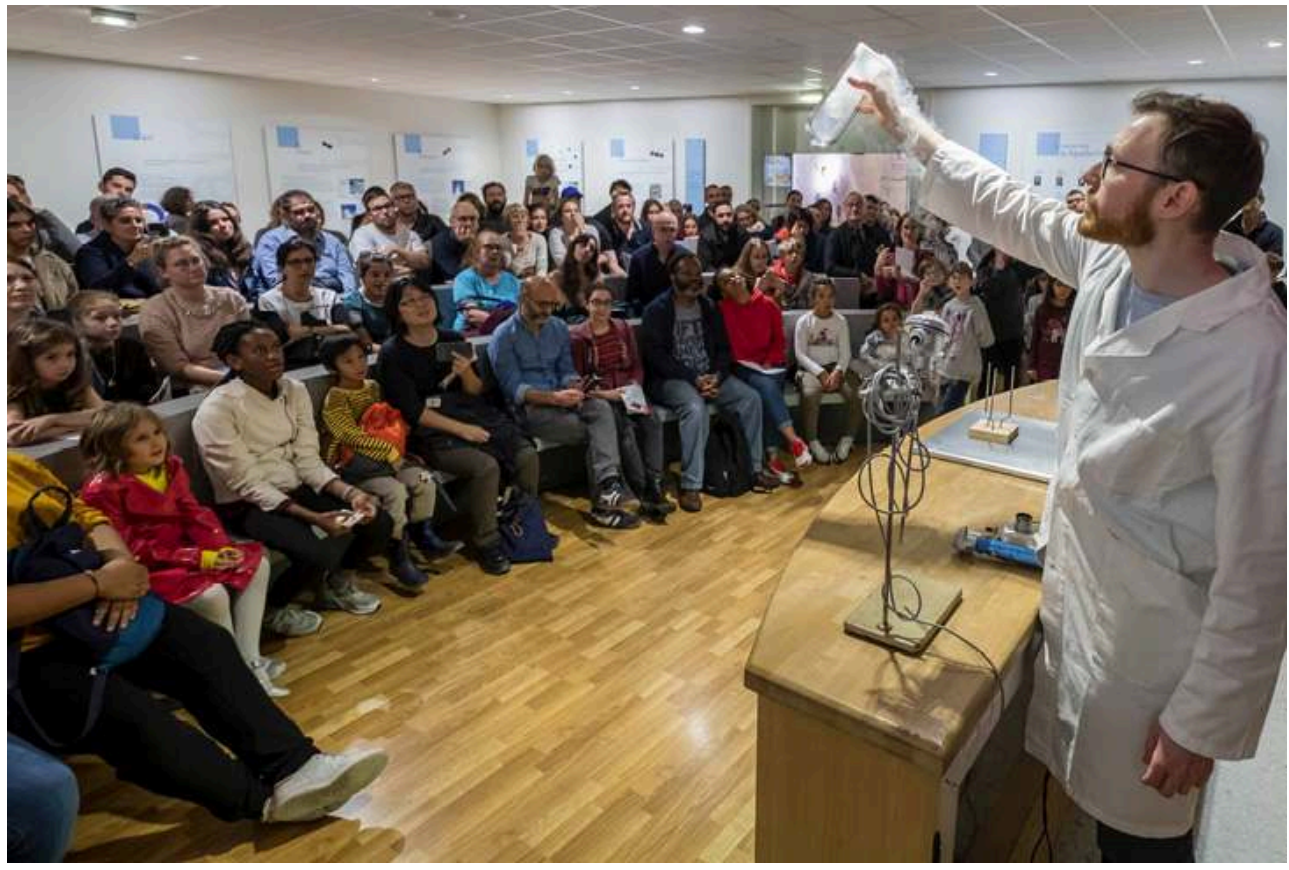

(c) A. Robin/EPPDCSI

7 Ce renouvellement s'appuie sur deux partis-pris forts. Premièrement, une prise de distance avec le modèle dit " du déficit ", qui part du postulat que le manque d'intérêt $\mathrm{du}$ public pour les sciences résulte d'un manque initial de connaissances. Nos concitoyens ont aujourd'hui, grâce aux technologies numériques, un accès facilité à l'information et aux savoirs scientifiques, dont le volume augmente à une vitesse vertigineuse. Par une médiation adaptée, il est possible, en partant de cette situation, d'offrir à tout un chacun la possibilité de s'approprier des concepts scientifiques 
complexes, de répondre lui-même aux questions qu'il se pose et de devenir un acteur responsable. Au principe descendant "enseigner-apprendre", se substitueront de nouvelles formes d'interaction cognitive, le couple «inviter-interagir " incarnant l'esprit d'échange, d'écoute et de création. En second lieu, les thématiques scientifiques abordées au Palais devront refléter la recherche contemporaine. Force est de constater que certaines sciences, comme celle du vivant par exemple, connaissent une montée en puissance; que de nouvelles disciplines, comme les sciences du numérique, se structurent $^{7}$; que les approches interdisciplinaires se développent, dans les laboratoires comme dans les amphithéâtres. Il est donc primordial que le Palais de la découverte rende compte de la scène scientifique d'aujourd'hui et soit conçu de manière à pouvoir rendre compte des évolutions futures.

La médiation humaine, résolument placée au cœur de la proposition pour le Palais de la découverte 2024, évoluera aussi pour s'adapter aux pratiques, aux usages, aux attentes non plus du public, mais des publics ${ }^{8}$.

Si les expériences spectaculaires ${ }^{9}$, les exposés et ateliers basés sur l'expérimentation resteront centraux, d'autres approches complémentaires seront développées, tirant parti de l'expérience acquise par les acteurs de l'enseignement des sciences ou de la communication scientifique en France et à l'étranger. Un centre de recherche sur la médiation scientifique sera créé, afin d'appliquer à nos métiers la méthode scientifique et de les faire progresser grâce à la science. Sans attendre, un incubateur a été mis sur pied, permettant de tester de nouvelles formes d'intervention auprès des visiteurs et privilégiant la dimension de co-construction avec ces derniers.

10 La technologie numérique sera mise à contribution selon une approche raisonnée. Il ne s'agit pas d'être pour (au nom du principe selon lequel les jeunes générations sont "numériquement natives») ou d'être contre (les écrans étant partout, les musées doivent s'en prémunir pour cultiver leur singularité ${ }^{10}$ ): les écrans ou les lunettes de réalité virtuelle sont des outils au service de la médiation humaine; leur utilisation n'est pas une fin en soi, la relation humaine demeurant la clé de voûte du musée sans collection ${ }^{11}$ qu'est le Palais de la découverte. On citera pour exemple des réalisations à venir dans ce domaine la création d'un espace immersif pouvant diffuser en direct ou en différé des flux d'images en haute définition en provenance des fonds marins, de la canopée amazonienne, des pentes d'un volcan ou encore d'un bloc opératoire... Les médiateurs pourront se saisir de ces images et guider l'interaction des publics avec les scientifiques en action, pour un décryptage en direct de la science en train de se faire.

11 La mue de l'institution concerne également les publics auxquels elle entend s'adresser. Bien entendu, le Palais de la découverte 2024 aura toujours la volonté de s'adresser au plus large public, en étant le plus inclusif possible, dans le respect de la singularité de chacun.

Il apparait toutefois souhaitable d'accorder une place particulière au public adulte, trop rarement considéré par les musées de science comme un groupe à part entière et qui se trouve souvent face à une offre pour le jeune public éloignée de ses besoins. Or la relation des adultes à la science est fondamentale dans nos sociétés contemporaines : les débats de société auxquels ils prennent part sont souvent de nature technoscientifique ; leurs impôts financent la recherche publique; citoyens éclairés, ils sont en mesure de modifier leurs habitudes de vie et de consommation, pour mieux contribuer à la préservation de l'environnement. 


\section{L'incubateur du Palais de la découverte}

L'incubateur est une démarche participative qui ambitionne d'inclure les publics experts dans la conception des offres, depuis l'idée jusqu'à leur mise en forme : des personnes en situation de handicap pour travailler le design pour tous, des enseignants pour bâtir une médiation à destination des élèves, des agronomes pour élaborer un atelier présenté au laboratoire du vivant, etc.

Médiateurs, muséographes et autres professionnels de la communication scientifique pourront ainsi concevoir, puis tester de nouveaux types de dispositifs pour s'adresser au public. Travaillant avec les groupes cibles dans différents formats (focus groups, ateliers, hackathons, etc.), ils disposeront des ressources matérielles et méthodologiques du Fablab et du Livinglab, mettant ainsi à profit les apports de la démarche de design collaboratif. L'incubateur ouvrira au sein du Palais de la découverte de juin à août 2020 pour une première expérimentation. Proposition émanant des médiateurs de l'établissement, son objectif est de permettre aux visiteurs d'être, d'ores et déjà, partie prenante du projet du futur Palais.

Le futur Palais de la découverte, co-construit avec la communauté scientifique et ses publics, sera un lieu ouvert, perméable à ce qui se passe dans le monde et dans la science. Il entend devenir un point de repère, un lieu de rencontres, une enceinte adaptée au débat, un espace pour le développement de projets à destination de la plus large diversité de communautés. Encore plus qu'aujourd'hui, les chercheurs, et notamment les jeunes chercheurs, y trouveront un lieu de présentation de leurs travaux, de rencontres avec leurs collègues, de formation à la médiation scientifique et de dialogue avec les publics: le Palais contribuera ainsi, à l'instar de l'ensemble des institutions de culture scientifique, à augmenter la "surface de contact» entre les scientifiques et la société civile. Les enseignants, avec l'aide des médiateurs, viendront au Palais de la découverte prolonger leurs cours, les enrichir et y développer des approches pédagogiques innovantes, fondées notamment sur l'expérimentation. Les élus, les associations ou les ONG, pour leur part, seront assurés de trouver au Palais de la découverte une information scientifique fiable et les ressources nécessaires pour interagir avec la science contemporaine. 
Vue d'un espace intérieur dédié à la biologie dans le futur Palais de la découverte " éphémère ».

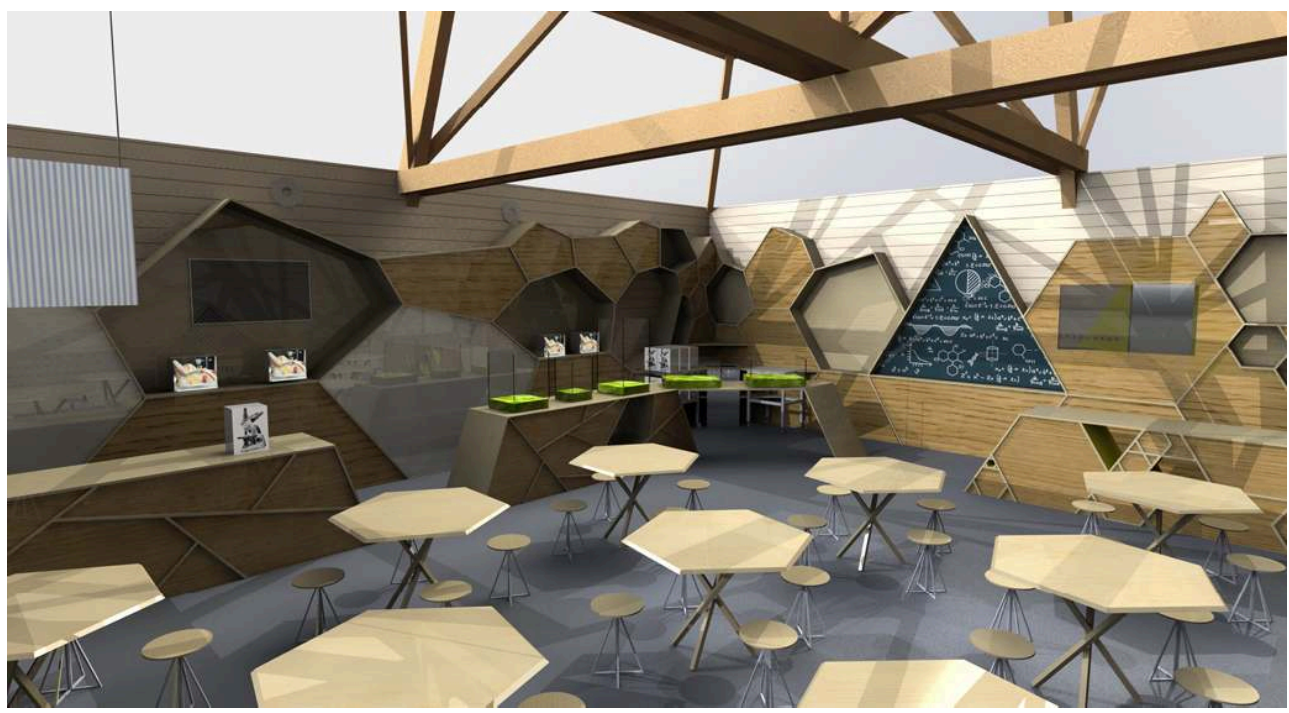

(c) EPPDCSI global du Grand Palais est un formidable stimulant pour développer la dimension artscience présente depuis les origines, l'État ayant passé de nombreuses commandes à des artistes pour l'ouverture en $1937^{12}$; une dimension longtemps incarnée par André Leveillé, peintre et premier directeur du Palais de la découverte ${ }^{13}$. Art et science constituent deux manifestations distinctes du génie créatif humain; elles servent complémentairement à interroger le monde et l'époque. Faire dialoguer ces approches ${ }^{14}$ confère une puissance particulière aux questionnements scientifiques, par le détour sensible que cela ménage. Cette ambition est à la base de la future galerie des enfants, nouvelle proposition "art-science» à destination du très jeune public, actuellement non servi par l'offre du Palais. Elle se manifestera également par des commandes à des artistes émergents, à charge pour eux de travailler avec de jeunes scientifiques, pour traiter, par exemple, l'épigénétique ou la matière noire, instaurant une tension signifiante entre les découvertes du passé et les défis de la recherche contemporaine.

\section{son bâtiment restauré.}

Ce temps, qui sera inauguré fin août par un Entracte au Palais, sera consacré à développer les futurs contenus scientifiques, en collaboration étroite avec la communauté scientifique, le projet scientifique et culturel en constituant le squelette conceptuel. Une exposition itinérante sur l'esprit critique, conçue avec les centres de culture scientifique de Bordeaux et de Toulouse, fera voyager en France le Palais de la découverte.

Enfin, l'institution prendra à l'automne ses quartiers dans le 15e arrondissement, au sein d'une structure éphémère accueillant des salles de médiation et un planétarium. Ce faisant, le Palais de la découverte maintiendra vivant le contact avec le public, sans lequel il n'est rien. 


\section{NOTES}

1. Présentation $\mathrm{du}$ projet sur le site $\mathrm{du}$ cabinet Chatillon Architectes : www.chatillonarchitectes.com/projets/le-grand-palais

2. L'opération est portée par la RMN-Grand Palais, maître d'ouvrage, et l'Opérateur du patrimoine et des projets immobiliers de la Culture, maître d'ouvrage délégué. Deux maîtres d'œuvre sont en charge l'un de la restauration (l'agence François Châtillon architecte, responsable du bâtiment en tant qu'architecte en chef des monuments historiques), l'autre de l'aménagement (l'agence LAN, désignée en février 2014 lauréate du concours). Cette dernière, qui vient de livrer le théâtre du Maillon à Strasbourg, a restauré les serres du Jardin des plantes à Paris. Universcience est associé à la maitrise d'ouvrage.

3. Sa synthèse est accessible ici: www.palais-decouverte.fr/fileadmin/fileadmin_Palais/ fichiersContribs/au-programme/evenements/nouveau-palais/Projet-scientifique-et-culturelPDLD-19-juin-2019.pdf

4. Le Palais de la découverte retrouvera son lustre originel grâce à la restauration des décors 1900 qui n'ont pas été détruits au cours du temps, à la différence d'autres parties du Grand Palais. Il bénéficiera également de zones de circulation agrandies et de services, tels qu'attendus dans un établissement $\mathrm{du} \mathrm{XXI}^{\mathrm{e}}$ siècle. Les groupes, nombreux à être accueillis par le Palais de la découverte, et notamment scolaires, bénéficieront d'une entrée spécifique. Enfin, l'accessibilité pour les personnes souffrant de handicaps sera conforme aux normes contemporaines.

5. Aux salles réservées à une discipline, succèderont des espaces, en nombre inférieur, se prêtant à des activités plus variées tout au long de la journée. Cette nouvelle organisation spatiale trouvera sa première traduction dans le Palais éphémère, dont il sera question plus loin.

6. Catalogue du Palais de la découverte, 1938.

7. Le Palais de la découverte s'est ainsi doté en 2016 d'une nouvelle unité scientifique et en 2018 d'un nouvel espace permanent d'exposition consacrés à l'informatique et aux sciences du numérique.

8. Il s'agira de ne plus prendre les visiteurs comme un tout indistinct, mais de différencier autant que faire se peut les propositions en fonction des différentes catégories de public. Le Palais de la découverte, outre son orientation traditionnelle envers la jeunesse qu'il entend cultiver, développera ainsi et par exemple, des activités spécifiquement destinées aux adultes (cf. infra).

9. Chaque discipline bénéficiera demain (ce qui n'est pas le cas aujourd'hui) d'une démonstration spectaculaire de référence, les « icônes ", selon la terminologie du projet scientifique et culturel. La manipulation d'électrostatique se retrouvera bien évidemment dans le Palais de 2024 et continuera de faire se dresser les cheveux sur la tête (pour le plus grand bonheur des visiteurs).

10. Ce faisant, le Palais de la découverte renoue avec l'esprit d'innovation de ses fondateurs. On se rappellera que Jean Painlevé, inventeur du cinéma scientifique, l'introduisit au Palais dès ses débuts.

11. Sans collection, mais non sans présentations permanentes, appelées "ilots de curiosité ». Sans collection, mais non sans objets, dont l'inventaire, mené avec l'aide d'une commission d'experts extérieurs, vise à identifier ceux présentant un caractère patrimonial.

12. À Fernand Léger notamment, ou encore à Marcel Gromaire dont les œuvres viennent d'être restaurées.

13. De 1937 à 1960.

14. Le Palais de la découverte participe depuis 2016 à la Foire internationale d'art contemporain de Paris, notamment en prenant part à Parades for FIAC, programmation en spectacle vivant. 


\section{RÉSUMÉS}

Le Palais de la découverte, lieu emblématique et historique de la culture scientifique et technique fera bientôt l'objet d'une rénovation attendue. Bruno Maquart, président d'Universcience, revient sur le contexte, les étapes et les axes forts du projet.

INDEX

Mots-clés : rénovation muséale, centre de sciences, CCSTI

\section{AUTEUR}

\section{BRUNO MAQUART}

Président d'Universcience (Palais de la découverte, Cité des sciences et de l'industrie)

bruno.maquart@universcience.fr 